\title{
FRACTAL ANALYSIS OF PATHFINDING AESTHETICS
}

\author{
Coleman, R. \\ Computer Science Department, Marist College, Poughkeepsie, New York 12601, United States \\ E-Mail: ron.coleman@marist.edu
}

\begin{abstract}
We study a new, fractal-based model of pathfinding aesthetics for videogames and other virtual worlds. This model fills a gap in previous pathfinding efforts that have studied mostly machine performance issues or relied on anecdotal arguments rather than metrics to hypothesize about and improve aesthetic outcomes. We show firstly that the fractal model consistently discriminates between paths that were generated with beautifying treatments versus control paths. We also report that the model reliably predicts player expectations of relative aesthetic values for pathfinding. These conclusions are supported by statistical analysis of model results and opinion survey responses.

(Received in August 2007, accepted in January 2008. This paper was with the author 2 months for 2 revisions.)
\end{abstract}

Key Words: Fractal Dimension, Pathfinding, Aesthetics, Virtual Worlds, Beautifying Heuristics

\section{INTRODUCTION}

Pathfinding in videogames enables allies, enemies, and other autonomous, simulated agents to navigate virtual worlds. Much of the literature on artificially intelligent pathfinding has focused on machine efficiency and exploring the search space of shortest paths. From the player's perspective, however, these concerns may be secondary or even undesirable if they generate unrealistic and mechanical-looking simulations [1]. Paths also have sensoriemotional or aesthetic qualities that must appeal to player expectations as plausible and natural, e.g., what natural intelligence might conceivably do.

Yet, aesthetics in general and beauty in particular, real or simulated, are thought to be subjective and relative. The philosopher, Immanuel Kant (1724-1804), argued in The Critique of Judgment [2] that beauty is not an inherent property of objects but a matter of taste. Indeed, game researchers and developers have approached the problem not by asking what is beauty in pathfinding but what is knowable about such beauty which can be captured by pathfinding heuristics called "aesthetic corrections" and "aesthetic optimizations." These investigators have nevertheless relied on anecdotal arguments, rather than metrics, to hypothesize about and test the efficacy of different beautifying treatments to improve aesthetic outcomes.

In this paper, we study a new method of measuring pathfinding aesthetics based on fractal analysis [3]. While fractal analysis has been used by others examining masterpieces and other works of art to assess aesthetic values, this approach has not been previously applied to pathfinding. In this case, we say a path object, $P_{1}$, has more "fractal beauty" than a reference path object, $P_{0}$, if $G\left(P_{1} \mid P_{0}\right)>0$ where $G$ is the fractal model which is subject to constraints we describe later.

We show in a series of experiments of randomly generated, multi-room virtual worlds that $G>0$ consistently with $p \approx 10^{-76}$ when $P_{1}$ has been generated with beautifying heuristics and $P_{0}$ is the reference or control. We report furthermore that these $P_{1}$ objects seem to reliably comport with and predict player expectations of relative beauty, etc., with $p \approx 10^{-15}$ according opinion survey data. 


\section{BACKGROUND AND RELATED WORK}

The literature contains many references on generating aesthetic shapes for simulation, design styling, and other purposes. See Prusinkiewicz and Lindenmayer [4], Podehl [5], the FIORESII project [6], and Shroeder [3]. However, we are studying a related but different problem, not creating aesthetic effects but mathematically measuring what is known about them. Rockmore [7] gives a brief history going back to the late $19^{\text {th }}$ century of similar mathematical efforts related to the study of literary works. In 1930s, Birkhoff used his "theory of aesthetic value" [3] to quantify concepts like "pleasing," "interesting," etc. found in music. However, Birkhoff was exploring stochastic processes characterized by fractional noise [3] which is related to the fractional dimension through a linear formulation [8].

Mandelbot [9] used the fractional dimension in his seminal paper to describe, among other things, irregularly shaped, finite length objects like coastlines. Others have since used the fractional (or fractal) dimension in a similar manner to analyze works of art like Bach's Brandenburg Concertos [3] and the "action paintings" of Jackson Pollack [7]. We use the fractal dimension similarly except to analyze the aesthetic content of experimental virtual world pathfinding.

Much of the literature on artificially intelligent pathfinding is silent on aesthetics. As examples, Russell and Norvig [10], Millington [11], and Seeman and Burg [12] provide thorough coverage of automated movement and pathfinding but they do not address aesthetics.

Some investigators have nevertheless recognized this problem and proposed heuristics to make paths more "appealing," "pleasing," "realistic," etc. In practical terms these heuristics imply 1) smoothing and straightening paths and 2) avoiding obstacles without tracking them. However, Higgins [1] detects non-straight path segments in order to correct them but he does not score the results or suggest how to do so. Similarly, Rabin [13] identifies a number of methods as does Pinter [14] to improve aesthetic outcomes but these authors do not grade the results as such. Botea, et al, [15] find they have to resort to cleaning up and beautifying the results of hierarchical pathfinding but they do not measure how much they improved the solution quality. Stout [16] faces an analogous situation by considering pathfinding in "smooth worlds" without actually using those criteria to define or rank outcomes.

Coleman [17] incorporated just these ideas in a simple, non-linear model called the beauty intensity, $\Re$, which measures relative smoothness and wall tracking. That is, $R=k \log \left(I_{1} / I_{2}\right)$ where $k$ is a constant, $I_{1}=r\left(P_{1}\right), I_{2}=r\left(P_{2}\right)$, and $r(P)=(|P|-u(P)) /|P|$. The quantity $|P|$ is the length of a path object, $P$, and $u(P)$ is the detracting or "ugly duckling" index which is a weighted sum of zigzags and wall tracking. Thus, a path object, $P_{1}$, is said to have more "working beauty" than a reference path object, $P_{0}$, provided $\mathfrak{R}\left(P_{1} \mid P_{0}\right)>0$.

While $R$ was shown to consistently discriminate between paths treated with beautifying heuristics and those without such treatments, there are some $P$ for which the assumptions of $u(P)$ will be invalid and $\Re$ fails. For instance, if $u(P) \geq|P|$ then there will be negative values in the log formulation which is undefined mathematically. Such a condition may arise, for instance, if the weights used by $u(P)$ are chosen improperly. Furthermore, although $\mathfrak{R}$ was designed to conform to Weber-Fechner laws, $\mathfrak{R}$ values are not readily intuitive since they don't correspond directly to sensory data.

The fractal dimension is not hampered by these limitations. Firstly, anyone who has sensed texture or differences in texture understands intuitively what the fractal dimension measures and the meaning of differences thereof. Secondly, the fractal dimension makes no pathfinding assumptions. Consequently, it has potential to be statistically more robust. There is not, however, one interpretation of the fractal dimension but many that fall into two general 
categories: geometric and stochastic [8]. The former is based on the concept of yardsticks and the latter, Brownian fluctuations.

We use a geometric interpretation of the fractal dimension based on reticular cell counting, or the box counting dimension. We chose this method for two reasons. Firstly, the box counting dimension is conceptually and computationally straightforward. Secondly, there are high quality implementations we discuss later to reliably calculate the box counting dimension.

\section{FRACTAL DIMENSION}

Mandelbrot [18] described fractals as geometric objects which are no-where differentiable (i.e., textured) and self-similar at many scales. By "self-similar" Mandelbrot meant "each portion can be considered a reduced-scale image of the whole.” [19] Mandelbrot suggested that fractals offered a better description of shapes found in nature but such descriptions were only useful as first-order approximations.

Mandelbrot also said fractal objects have fractional dimension, $D$, namely, a non-whole number called the fractal dimension [9]. Mathematically, it is given by the Hausdorff dimension [3]:

$$
D(S)=\lim _{\varepsilon \rightarrow 0} \frac{\log N_{\varepsilon}(S)}{\log (1 / \varepsilon)}
$$

where $S$ represents a set of points of a surface (e.g., coastlines, brush strokes, paths, etc.), $\varepsilon$ is the size of the measuring tool or ruler and $N_{\varepsilon}(S)$ is the number of self-similar objects or subcomponents covered by the tool [3]. For fractal objects, $\log N_{\delta}(S)$ will be greater than $\log (1 / \varepsilon)$ by a fractional amount. If the tool is a uniform grid of square cells, then a straight line passes through twice as many cells if the cell length is reduced by a factor of two. A fractal object passes through more than twice as many cells.

Consider the coastline map of the state of Maine, United States, in the figure below. It is overlaid by an imaginary, uniform grid of larger (solid) and smaller (dash) square cells. The smaller cells are one-half the length of the larger cells. One can clearly see in several places where the coastline passes through more than twice as many smaller cells for a given larger cell. Maine's coastline is fractal accordingly.

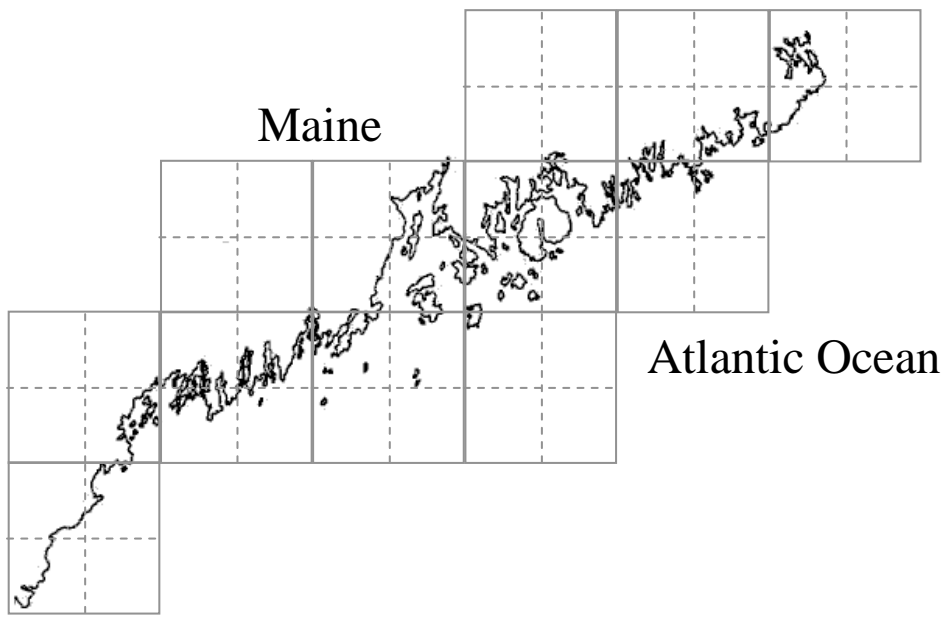

Figure 1: Maine, U.S., Atlantic coastline [20].

If the topological dimension of an object is the Euclidean or metric space an object inhabits, the fractal dimension also exceeds the object's topological dimension, $n$, namely, 
$n<D<n+1$. As an example, the Atlantic coastline of Maine is topologically one dimensional when viewed on a map. Yet, Maine's coastline zigzags and turns with many inlets, outcrops and keys. Researchers have estimated the fractal dimension to be between 1.11 and 1.37, depending on where measurements are taken [21].

For self-similar surfaces and a cell of length $\varepsilon$, the number of cells intersecting the surface is:

$$
N_{\varepsilon} \propto \varepsilon^{-D}
$$

According to Voss [8], if $P R_{S}(m, \varepsilon)$ is the probability of finding $m$ points in a particular cell, then the number of cells needed to cover the surface, $S$, of the entire image is:

$$
N_{\varepsilon}[S]=\sum_{m=1}^{\varepsilon \times \varepsilon} P R_{S}(m, \varepsilon)
$$

In general, the box-counting dimension is the rate at which the proportion of cells intersected by the surface increases as the cell size decreases. The Gagnepain algorithm [22] computes the fractal dimension, $D$, for a grey scale image per the following pseudo-code:

\section{Begin}

Set $N[\varepsilon] \leftarrow 0$ for all values of $\varepsilon$

For each pixel, $S(x, y)$, in the image

$m \leftarrow 0$

For each cell of size $\varepsilon$

Center a cell of size $\varepsilon$ on $(x, y)$

End Do

$$
m \leftarrow 1 \text { if } S(x-\varepsilon / 2, y-\varepsilon / 2) \text { to } S(x+\varepsilon / 2, y+\varepsilon / 2) \neq 0
$$

\section{End For}

If $m=1$, then increment $N[\varepsilon]$ by 1

\section{End}

Estimate $D$ as the regression slope of $\log (\varepsilon)$ vs. $\log (1 / N(\varepsilon))$

Jelinek, et al, [23] have implemented a version of this algorithm as part of the FracTop project distributed as a Java ${ }^{\mathrm{TM}}$ archive or jar [24]. FracTop takes the test image in PNG [25] format and computes $D$ using default cell sizes. FracTop also handles boundary conditions, for instance, if the image size is not a multiple of the cell size.

\section{MEASUREMENT MODEL}

Let $W$ be a finite state-space in Euclidean $R^{n}$ called a virtual world. (We assume $n=2$ but this is not strictly necessary.) Let $W: x, y \rightarrow t$ for $0 \leq x<w, 0 \leq y<h$, where $w$ and $h$ are width and height features respectively of $W$, and $t \in\{0,1,2\}$ is a set of states. Let $A$ be a simulated rigid-body with configurations (or steps) $A^{i}=W: A^{i} . x, A^{i} . y$ for $i=0 \ldots l$. Assume $A$ is "free flying" in that $A$ has potential configurations:

$$
\begin{aligned}
& A^{i+1} \cdot x=A^{i} \cdot x+\Delta x \\
& A^{i+1} \cdot y=A^{i} \cdot y+\Delta y
\end{aligned}
$$

provided $W: A^{i+1} . x, A^{i+1} . y \rightarrow 0$ for $\Delta x, \Delta y \in\{-1,0,1\}$. In other words, the admissible step transitions of $A$ are limited to the eight nearest neighbors. However, $A$ is further constrained by "stationary" objects, $\left\{B^{j}\right\}$, called obstacles, namely, $W: B^{j} \cdot x, B^{j} \cdot y \rightarrow 2$. 
The object, $P$, called a path, is an ordered sequence of steps, $\left[A^{\text {start }}, A^{1}, A^{2}, \ldots, A^{\text {goal }}\right]$ such that $W: A^{i} . x, A^{i} . y \rightarrow 1$ where start $=0$ and goal $>0$. Given $A^{\text {start }}$ and $A^{\text {goal }}$ as the initial and terminal steps of $P$, the pathfinding objective is to search for $A^{1}, A^{2}, \ldots A^{\text {goal-1 }}$, if they exist.

Let $D\left(P,\left\{B^{j}\right\}, W\right)$ be the fractal dimension of the 2D image implied by $P$ and $\left\{B^{j}\right\}$ in $W$. However, to simplify the notation, we use the shorthand $D(P)$ for $D\left(P,\left\{B^{j}\right\}, W\right)$ when it is clear what we mean. We define, $G$, the "fractal beauty" of a path, $P_{1}$, in relation to a reference path, $P_{0}$, as:

$$
G\left(P_{1} \mid P_{0}\right)=D\left(P_{1}\right)-D\left(P_{0}\right)
$$

This relation is meaningful only if $\left\{B^{j}\right\}, W, A^{\text {start }}$, and $A^{\text {goal }}$ are the same for both $P_{1}$ and $P_{0}$. Given these constraints, we say $P_{1}$ has more "fractal beauty" than $P_{0}$ only if:

$$
G\left(P_{1} \mid P_{0}\right)>0
$$

\section{EXPERIMENTAL METHODS}

From an experimental design perspective, the measurement model is a "black box." Namely, we input two objects, $P_{0}$ and $P_{1}$, and we get a result, a statistic, $G$. In the previous section, we described the inner workings of the model, that is, how it uses $\mathrm{P}_{0}$ and $\mathrm{P}_{1}$. Yet, the experimental design analyzes only model results. It does not ask how the model internally derives results, how it computes and uses the fractal dimensions, indeed, that fractals are involved at all. The experimental design is deliberately blind to these operations. For this reason, as we describe later, we use nonparametric tests as they generally make the fewest assumptions about $G$ per the null hypothesis which we explain below.

The opinion survey is similar. Respondents are like black boxes as far as the experimental design is concerned. We provide each respondent with one or more pairs of path objects. Yet we do not ask how or why respondents reach their opinions - that aspect is not relevant to the experiment. We instead analyze responses per the null hypothesis for the same virtual worlds we give to the model. The opinion survey null hypothesis is also covered below.

\subsection{Virtual world simulations}

We use the same 100 virtual worlds from Coleman [17]. Each world is 50 tiles wide and 50 tiles deep. Each tile is 10 pixels square and each $A^{i}$ and $B^{j}$ occupy exactly one tile as a filled black circle and square, respectively. The obstables, $\left\{B^{j}\right\}$, form interconnected rooms and hallways created by the Wells [26] random level generator. It requires a seed to randomize the configuration of rooms and hallways and then, it initializes each world with $A^{\text {start }}$ and $A^{\text {goal }}$ in the first and last rooms, respectively.

To generate $P_{0}$, the control, we use the "standard" A* algorithm from Bourg and Seeman [12] with the Manhattan distance as the cost heuristic. It uses no beautifying methods. To generate $P_{1}$, we use the "aesthetic" $A^{*}$ algorithm from Coleman [17]. It searches for a path using Higgins- [1] and Rabin-type [13] beautifying heuristics.

We convert $P,\left\{B^{j}\right\}$, and $W$ to a PNG [26] image. Then, we configure FracTop [23] with the pixel cell sizes, $\{2,3,4,6,8,12,16,32,64,128\}$. These are the default cell sizes of FracTop. They also yield fit fractal dimensions, that is, the coefficient of determination, $R^{2}$, is approximately 0.99 on the data sets we study.

Finally, we invoke FracTop with the PNG image. FracTop returns the fractal dimension, including the regression data, which we store in a database for further analysis. We execute this process for $k=1-100$ times. Each $k$ is a virtual world containing randomly seeded $W$ and $\left\{B^{j}\right\}$. They are the same for $P_{0, k}$ and $P_{1, k}$ which represent the $k$-th control path generated by 
the standard $\mathrm{A}^{*}$ algorithm and the aesthetic $\mathrm{A}^{*}$ algorithm, respectively. We retrieve the fractal statistics from the database and compute $G_{k}\left(P_{1, k} \mid P_{0, k}\right)$ using equation (6) for each $k$ virtual world.

We use the one-tailed sign test [28] which makes few assumptions about the distribution of $G$ and so the sign test is very reliable but not powerful. The sign test is also simple to compute and readily suitable for equation (7). That is, we have model observations, $G_{k}\left(\mathrm{P}_{1, \mathrm{k}} \mid\right.$ $\left.\mathrm{P}_{0, \mathrm{k}}\right)$, for $k=1-100$. The null hypothesis is $H_{0}: E\left[G_{k}\left(P_{1, k} \mid P_{0, k}\right)\right] \leq 0$. The alternative is $H_{1}: E$ $\left[G_{k}\left(P_{1, k} \mid P_{0, k}\right)\right]>0$. T is the frequency count of $G_{k}\left(P_{1, k} \mid P_{0, k}\right)>0$. We reject $H_{0}$ if $T>N-t$ where $N=100 ; t=1 / 2\left(N+w_{\alpha} \times \sqrt{ } N\right)$; and $w_{\alpha}$ is the critical region for a $p$-value of $\alpha$. The $p$ value and $\alpha$ are chosen as the smallest value for which $T$ is statistically significant.

We also use the Kolmogorov-Smirnov test [28] to examine the normality of $G$. The null hypothesis is that the distribution is normal. In that case, if $G$ is normal, we will use a more powerful test, like the t-test [27], in addition to the sign test to assess the statistical significance of $G$.

\subsection{Opinion Survey}

We regenerate the same 100 pairs of virtual world images but this time to embed them in opinion survey forms. The difference is we add labels to the start and goal configurations and we change the color of each $A^{i}$ to make the paths more visually distinguishable as the reader can see in the sample form for Trial 1 in the figure below. The paths through this multi-room virtual world are shown by the "bread crumbs."

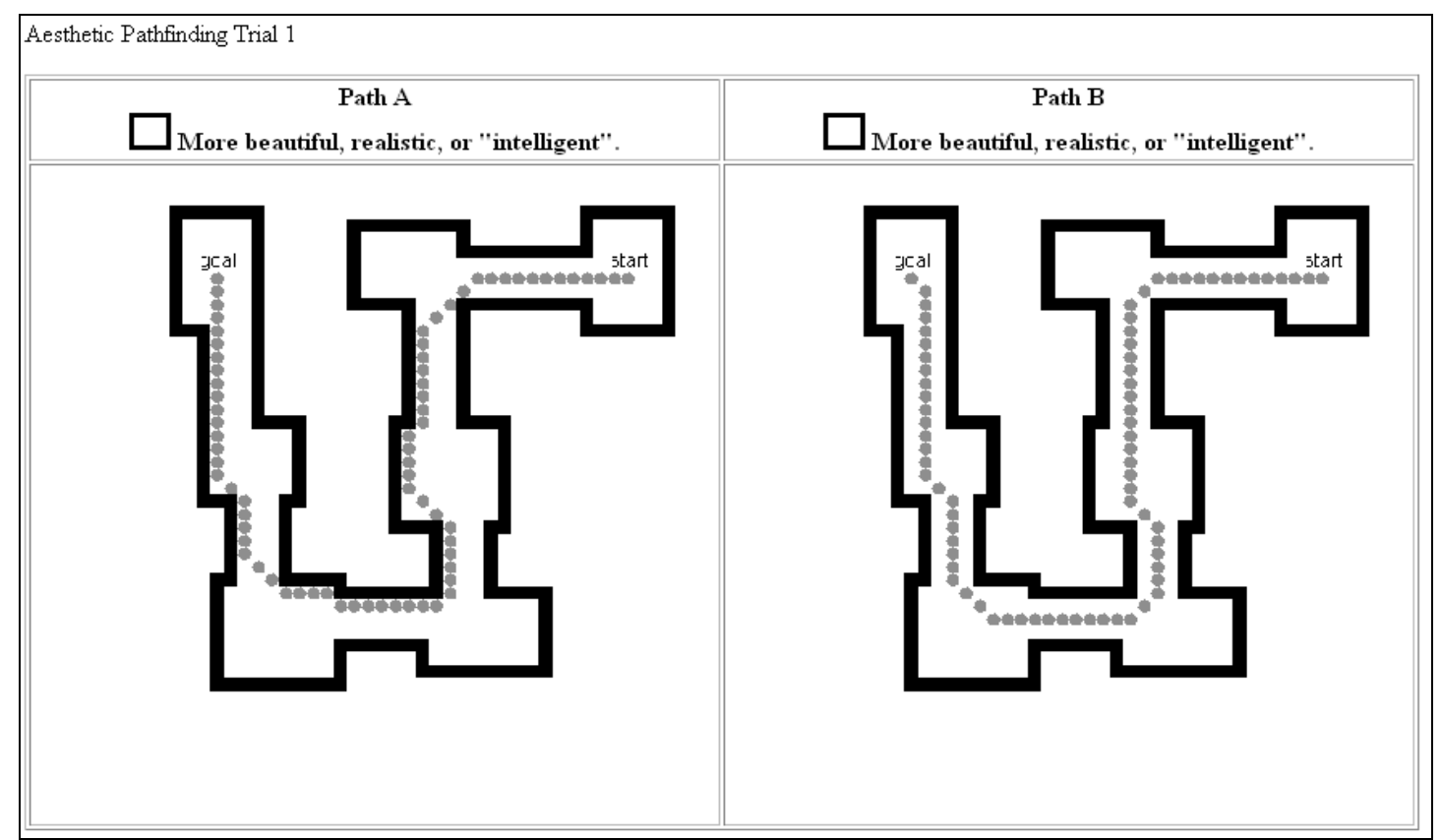

Figure 2: A sample opinion survey form for the random level, Trial 1, shown in an overhead, 2D view.

Respondents were asked to check the box for Path A or Path B. In the above case, Path A is the control and Path B is the aesthetic. On different forms, the control and aesthetic paths were randomly swapped. We administered this survey during our game design and programming class of 25 undergraduate and graduate students who were self-described videogame players. Prior to issuing the survey, students were also given an introduction to the subject of pathfinding. The complete survey of random, multi-room virtual worlds can be downloaded at [29]. 
To estimate the $p$-value of survey responses, we use the one-tailed Binomial test [27], a standard nonparametric test. That is, we treat each response form as an independent Bernoulli trial. The number of successes, $s$, is the number of trials in which the respondent selects the aesthetic path as "more beautiful, realistic, or 'intelligent"'. The null hypothesis is $H_{0}: s \leq 50$. The alternative is $H_{1}: s>50$. The probability of success is $q=0.50$. The $p$-value is one minus the cumulative Binomial distribution for $s$ successes in 100 trials.

\section{AN EXAMPLE}

In the interest of making these concepts clearer, we go through the details of an example, namely, Trial 1 in the figure above. Path $\mathrm{A}$ and Path $\mathrm{B}$ are $P_{0}$ (control) and $P_{1}$ (aesthetic), respectively. The $\log (\varepsilon)$ vs. $\log (1 / N(S))$ data for the control and aesthetic paths are in the table below. Note the aesthetic path is intersecting more cells for all grids except the two largest ones, $\varepsilon=64$ and $\varepsilon=128$ pixels, where the aesthetic and control paths are the same.

Table I: Cell length, $\varepsilon$, and cell count, $N(S)$, for control v. aesthetic paths.

\begin{tabular}{|r|r|r|r|r|r|}
\hline & \multirow{2}{*}{$\varepsilon$} & \multicolumn{2}{|c|}{ Control $\left(P_{0}\right)$} & \multicolumn{2}{c|}{ Aesthetic $\left(P_{1}\right)$} \\
\cline { 3 - 6 } & $\log (\varepsilon)$ & $N(S)$ & $\log (1 / N(S))$ & $N(S)$ & $\log (1 / N(S))$ \\
\hline 2 & 0.693147 & 7212 & -8.88350 & 7287 & -8.89385 \\
\hline 3 & 1.098612 & 3846 & -8.25479 & 3945 & -8.28020 \\
\hline 4 & 1.386294 & 2269 & -7.72709 & 2362 & -7.76726 \\
\hline 6 & 1.791759 & 1141 & -7.03966 & 1249 & -7.13010 \\
\hline 8 & 2.079442 & 745 & -6.61338 & 811 & -6.69827 \\
\hline 12 & 2.484907 & 377 & -5.93225 & 428 & -6.05912 \\
\hline 16 & 2.772589 & 275 & -5.61677 & 292 & -5.67675 \\
\hline 32 & 3.465736 & 99 & -4.59512 & 100 & -4.60517 \\
\hline 64 & 4.158883 & 30 & -3.40120 & 30 & -3.40120 \\
\hline 128 & 4.852030 & 8 & -2.07944 & 8 & -2.07944 \\
\hline
\end{tabular}

The figure below shows the corresponding scatter plot for control (“ $\triangle$ ”) and aesthetic paths (“+”).

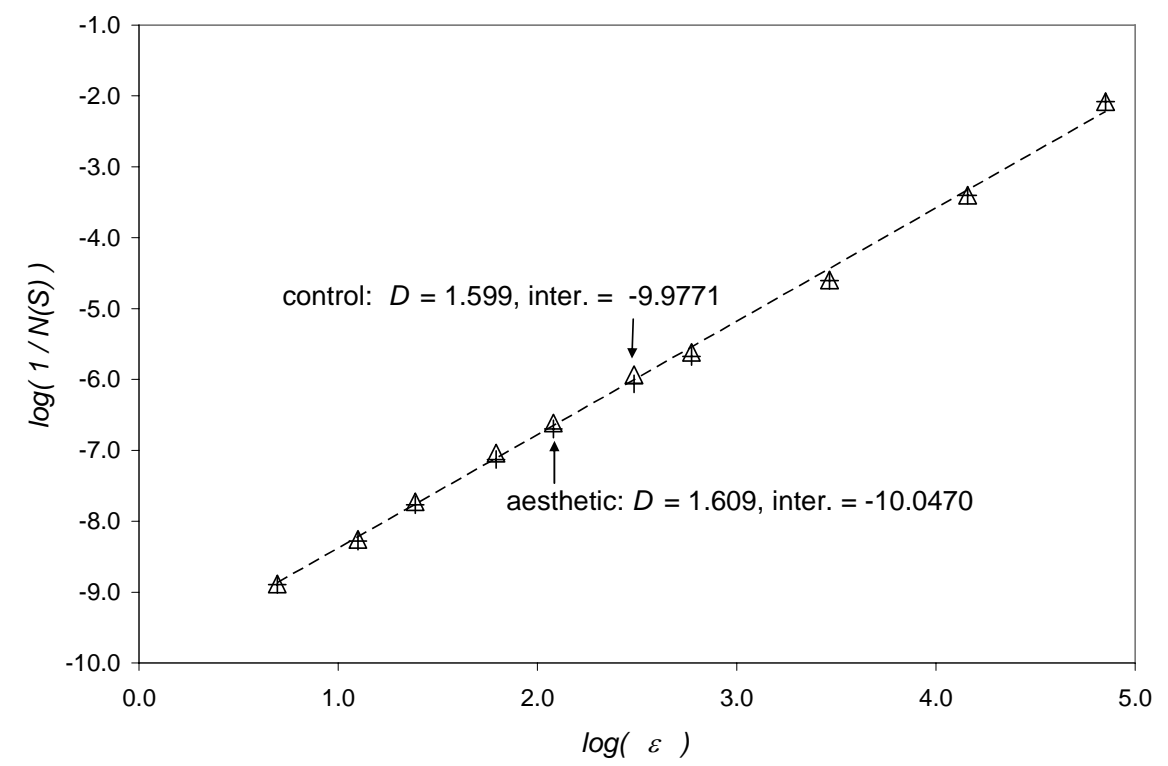

Figure 3: The scatter plot and regression of $\log (\varepsilon)$ vs. $\log (1 / N(S))$ for both control and aesthetic paths. Arrows point to sample points from each data set. 
For this particular trial, $D\left(P_{0}\right)=1.5989$ and $D\left(P_{1}\right)=1.6090$. According to equation (6), $G=0.0101$. The aesthetic path, $P_{1}$, is thus said to have more fractal beauty in relation to the control, $P_{0}$.

\section{RESULTS}

The 100 trials give a mean $G$ equal to 0.010256 with standard deviation of 0.001857 . The figure below is the distribution histogram of $G$.

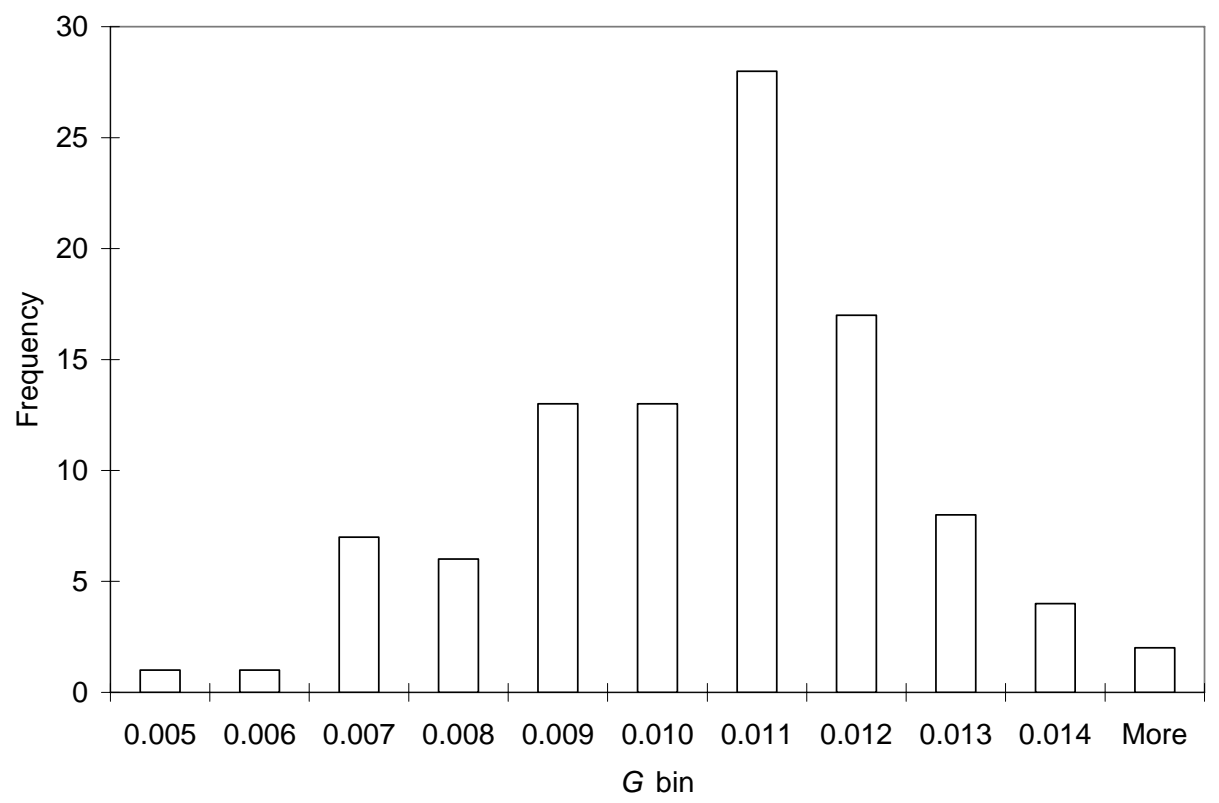

Figure 4: Histogram of $G$ from 100 virtual world experiments.

Since all the $G$ values are positive, $T=100$ according to the sign test. The critical region corresponds to $w_{\alpha}=-3.7190$ for $\alpha=0.0001$ and $N-t=100-1 / 2(100-3.7190 \times \sqrt{100})=62.81$. It happens that $\alpha=0.0001$ is the smallest value listed in table A3 of Conover [27] so the $p$-value is less than $10^{-4}$. For the opinion survey, respondents similarly selected the aesthetic path in every case. The $p$-value according to the Binomial test is approximately $10^{-15}$.

We invoked the Kolmogorov-Smirnov test to determine if the distribution shown in Fig. 4 is normal. The K-S statistic is 0.55 with $p \approx 10^{-14}$. In other words, the distribution appears to be normal or Gaussian. We invoked the t-test using Microsoft Excel ${ }^{\circledR}$ and found $p \approx 10^{-76}$.

Finally, we note that both $G$ and $\mathscr{R}$ make the same predictions in all 100 trials [17]. In other words, they confirm each other. Yet $G$ and $\Re$ are only mildly correlated $(r=0.22)$. Furthermore, the variance-to-mean ratio (VMR) for $G$ is 0.0003 . For $\mathfrak{R}$, the VMR is 0.0818 [17]. In other words, $G$ is a more reliable estimator of aesthetic appeal by more than two orders of magnitude and for this reason and others we mentioned earlier, we believe $G$ is a superior model.

\section{CONCLUSIONS}

We have shown that aesthetic paths consistently have higher fractal dimensions which comport with player expectations of plausibility, realism, etc. While the data indicates that model results vary in a relatively narrow range compared to the mean, we have not studied whether or under what conditions these variations correlate with aesthetic appeal. 
Our experimental analysis has furthermore focused mainly on pathfinding with specific aesthetic objectives, e.g., for single agents navigating by straight lines, smooth turns, and avoiding obstacles without tracking them. In some videogames, these objectives may be insufficient. Agents may move with different aesthetic objectives, for instance, for stealth or in groups like herds, armies, etc. The issue is not necessarily how to generate such movements but how to measure them in such a way that facilitates hypothesis formation and testing of aesthetic outcomes.

These and other considerations seem to us to be interesting topics for fractal analysis and future research.

\section{ACKNOWLEDGEMENTS}

The author thanks Matthew Johnson, Alan Labouseur, and Maria Coleman for reading drafts. The author is also grateful to the reviewers for insightful feedback to improve the paper.

\section{REFERENCES}

[1] Higgins, D. (2002). Pathfinding Design Architecture, AI Game Programming Wisdom, S. Rabin (ed.), Charles River, 114-121

[2] Kant, I. (1978). The Critique of Judgment, 1790, trans. Meredith, J.C., Oxford University Press

[3] Schroeder, M. (1992). Fractals, Chaos, and Power Laws, Freeman

[4] Pursinkiewicz, P.; Lindenmayer, A. (1990). The Algorithmic Beauty of Plants, Springer-Verlag

[5] Podehl, G. (2002). Terms and Measures for Styling Properties, International Design Conference - Design 2002, Dubrovnik

[6] Giannini, F.; Monti, M. An innovative approach to aesthetic design, http://www.fiores.com/ FIORES2/FIORES2.html, accessed on 2-12-2007

[7] Rockmore, D. (2006). The Style of Numbers Behind a Number of Style, The Chronicle of Higher Education, June 9, 2006

[8] Soille, P.; Rivest, J. G. (1996). On the Validity of Fractal Dimension Measurements in Image Analysis, Journal of Visual Communication and Image Representation, Vol. 7, 217-229

[9] Mandelbrot, B. (1967). How long is the coast of Britain? Statistical self-similarity and fractional dimension, Science, Vol. 156 (3775), 636-638

[10] Russell, S.; Norvig, P. (2003). Artificial Intelligence, Prentice Hall

[11] Millington, I. (2006). Artificial Intelligence for Games, Morgan Kaufman

[12] Bourg, D. M.; Seemann, G. (2004). AI for Game Developers, O’Reilly

[13] Rabin, S. (2000). Aesthetic Optimizations, AI Game Programming Gems, M. DeLoura (ed.), Charles River, 264-271

[14] Pinter, M. Toward More Realistic Pathfinding, http://www.gamasutra.com, accessed on 4-102006

[15] Botea, A.; Muller, M.; Schaeffer, J. (2004). Near Optimal Hierarchical Path-Finding, Journal of Game Development, Vol. 1, March Issue, 7-28

[16] Stout, B. Smart Moves: Intelligent Pathfinding, http://www.gamasutra.com, accessed on 22-092006

[17] Coleman, R. (2007). Operationally Aesthetic Pathfinding, Proceedings of the 2007 International Conference on Artificial Intelligence, Vol. 1, CSREA Press, 159-163

[18] Mandelbrot, B. (1982). Fractal Geometry of Nature, Freeman

[19] Oliver, D. (1992). Fractal Vision, Sams

[20] Maine: Maine and United States Map Links, http://www.netstate.com/states/maps/me_maps.htm, accessed on 11-07-2007

[21] Tanner, B. R.; Perfect, E.; Kelley, J. T. (2006). Fractal Analysis of Maine’s Glaciated Shoreline Tests Established Coastal Classification Scheme, Journal of Coastal Research, Vol. 22, No. 5, 1300-1304, doi:10.2112/05-0474R.1 
[22] Behery, G. M. (2006). Simulation of Fractal Dimension Evaluations, International Journal of Simulation and Modeling, Vol. 26, No. 2, 91-97

[23] Jelinek, H.; Cornforth, D.; Weymouth, L. FracTop v.0.3B, http://seal.tst.adfa.edu.au/ s3165516 /Fractop/, accessed on 31-12-2006

[24] Sun Microsystems, http://java.sun.com, accessed on 23-07-2007

[25] Portable Network Graphics, http://www.libpng.org/pub/png/, accessed on 23-07-2007

[26] Wells, M. (2004). J2ME Game Programming, Thompson

[27] Conover, W. J. (1980). Practical Nonparametric Statistics, $2^{\text {nd }}$ ed., Wiley

[28] Press, W. H.; Teukolsky, S. A. (Eds.) (2002). Numerical Recipes in $C++, 2^{\text {nd }}$ ed., Cambridge University Press

[29] Coleman, R. Fractal Analysis of Pathfinding Aesthetics Survey, http://foxweb.marist.edu/users /ron.coleman/fapasurvey/AestheticPathfinding100x.html, accessed on 23-08-2007 